\title{
ZEEP: Zonal based Energy Efficient Routing Protocol for HWSN
}

\author{
Muhammad Rizwan ${ }^{1, a}$, Muhammad S. Nisar ${ }^{2, b}$ and Hongbo Jiang ${ }^{1, c}$ \\ ${ }^{1}$ School of Electronic Information and Communications, HUST, Wuhan 430074, China; \\ ${ }^{2}$ Wuhan National Laboratory for Optoelectronics, HUST, Wuhan 430074, China. \\ a1201422058@hust.edu.cn, ' I201322185@hust.edu.cn, chongbojiang@hust.edu.cn
}

Keywords: Clustering, Cluster Head (CH), Stability Period, Network Lifetime, Sink.

\begin{abstract}
In Wireless sensor network (WSN), sensor nodes are usually deployed in the remote dense environment with limited energy resources in order to monitor a region for a long time. It makes energy conservation as most challenging issue for design and deployment of wireless sensor networks. In the energy conversation solutions, most of the researches have been conducted on energy efficient solutions regardless of the intra-cluster and inter-cluster short distance energy efficient communication. Transmission distance is proportional to energy cost. So, energy cost can be reduced by shortening the communication distance between the nodes. To reduce the intra-cluster and inter-cluster transmission distance, a novel Zonal based Energy Efficient Routing Protocol (ZEEP) for Heterogeneous WSN has been proposed in order to enhance the stability period and lifetime of the network. The simulation results indicate that proposed scheme enhanced the stability period, network lifetime and throughput than existing routing protocols.
\end{abstract}

\section{Introduction}

Wireless Sensor Networks (WSNs) have gained worldwide attention over the past few years. The development of smart sensors technology has been facilitated by Micro-Electro-Mechanical Systems technology. A WSN is comprised of a large number of distributed autonomous sensor nodes that are deployed to monitor a physical or environment conditions, such as weather, temperature or pollutants. The sensor nodes are capable to sense, gather and measure the information and forward the sensed data to sink via possible multihop communication. WSNs are used in healthcare monitoring, habitat monitoring and industrial process monitoring [1]. Heterogeneous WSNs have become more popular because it takes full advantage of heterogeneity in order to meet the demands of various applications which have been studied in recent literature [2].

One of the most crucial challenges in the organization of WSN is energy efficiency, stability period and enhancement in network life time because the sensor nodes are energy-constrained, limited storage capacity and low in computational power. In most applications, the battery of the sensor nodes cannot be replaced or recharged due to their dense deployment in the remote harsh environment with less or no human intervention. Because sensor nodes have limited power resources, so it is important to introduce the novel energy conservation schemes in order to extend the life time of WSNs [3].

Clustering is an important mechanism to address the critical challenges related to energy consumption, network lifetime and scalability. Clustering has been widely used in various sensor applications. Cluster based routing schemes are very helpful by reducing the cost of data aggregation and transmission of sensed data before transmitting to sink [4].

\section{Heterogeneous and Energy Consumption Model}

This model consists of $n$ number of sensor nodes randomly deployed in MxM square meter region. Sink is located in the middle of sensing area. There are two types of nodes with different energy i.e. normal and advance nodes. Advance nodes have equipped with more initial energy than normal nodes. Let $E_{0}$ is the initial energy of each normal node and $(1+\alpha) E_{0}$ is the initial energy of each advance node. Let $\mathrm{m}$ be the fraction of total number of sensor nodes $\mathrm{n}$ having $\alpha$ times more energy than normal 
node called advance nodes and the rest of $n^{*}(1-m)$ as normal nodes in the network [6]. Total energy of the system is increased by a factor $(1+\alpha \mathrm{m})$. Total initial energy $\mathrm{E}_{\text {total }}$ of HWSN model is given by:

$$
n \cdot E_{0}(1-m)+n \cdot m(1+\alpha) E_{0}=N(1+\alpha m) E_{0}
$$

In this work, we use the radio energy dissipation model similar to [5]. Free space and multipath fading channel are used in this model. The amount of energy required to transmit $\mathrm{L}$ bits message over a distance $\mathrm{d}$ from one node to another node is given by:

$$
E_{T x}(L, d)=\left\{\begin{array}{l}
L \times \text { Elect }+L \times E_{f s} \times d^{2} \text { if } d<d_{0} \\
L \times \text { Elect }+L \times E_{m p} \times d^{4} \text { if } d<d_{0}
\end{array}\right.
$$

Elect is the dissipated energy to run the transmitter or receiver. The parameters $E_{f s}$ and $E_{m p}$ is the amount of energy dissipation which depends upon the distance $\mathrm{d}_{0}$ which is given as $d_{0}=\sqrt{E_{f s} / E_{m p}}$. To receive the $L$ bits message, dissipated energy is equal to $E_{r x}(L)=L . E_{r x-e l e c}$.

\section{ZEEP: Zonal based Energy Efficient Routing Protocol}

In ZEEP, $\mathrm{n}$ sensor nodes are randomly dispersend in the $\mathrm{Mx} \mathrm{M}$ sensing region. The distance from node to $\mathrm{CH}$ or sink is $\leq \mathrm{d}_{0}$. The energy dissipated by $\mathrm{CH}$ and non-CH in a single round is defined as:

$$
\begin{aligned}
& E_{C H}=L . \text { Elect }\left(\frac{n}{k}-1\right)+L . E_{D A} \frac{n}{k}+L . \text { Elect }+L . E_{f S} d_{t o B S}^{2} \\
& E_{n o n C H}=L .\left(\text { Elect }+E_{f s} \times d_{t o C H}^{2}\right)
\end{aligned}
$$

Where $E_{D A}$ is defined as the energy required for data aggregation in a round, k is defined as number of clusters, $d_{t o C H}$ is defined as the average distance between nodes and $\mathrm{CH}$ and $d_{\text {toBs }}$ is defined as average distance between cluster head and base station. The total expended energy can be calculated as follows:

$$
E_{\text {total }}=L .\left(2 n E_{\text {elec }}+n E_{D A}+k E_{f S} d_{\text {toBS }}^{2} \times n E_{f S} d_{\text {toCH }}^{2}\right)
$$

The optimal number of clusters can be calculated by $k_{\text {opt }}=\sqrt{E_{f s} / E_{m p}} \times \sqrt{n / 2 \pi} \times \sqrt{M / d_{\text {toBs }}^{2}}$ the average distance $d_{t o B S}$ can be calculated as follows [6] $d_{t o B S}=0.765 \times M / 2$ and the optimal probability of node to become a $\mathrm{CH}$ in a round is defined as $p_{\text {opt }}=k_{\text {opt }} / n$. Let $p_{n r m l}, p_{a d v}$ represents the weighted election probability for normal and advance nodes. The average number of $\mathrm{CHs}$ per round per epoch is equal to $n .(1+\alpha m) p_{n r m l}$. The weighted probability of normal and advance nodes can be calculated as:

$$
p_{\text {nrml }}=\frac{p_{\text {opt }}}{1+\alpha . m}, p_{\text {adv }}=\frac{p_{\text {opt }}}{1+\alpha . m} \times(1+\alpha)
$$

In the two-level HWSN, we replace the reference $p_{\text {opt }}$ value with the weighted probabilities given in Eq. 6. Therefore, $p_{i}$ is changed into:

$$
p_{i}=\left\{\begin{array}{c}
\frac{p_{\text {opt }} E_{i}(\text { res })}{(1+\alpha \cdot m) \bar{E}(r e s)} \text { if } S_{i} \text { is normal node } \\
\frac{p_{\text {opt }} E_{i}(\text { res })(1+\alpha)}{(1+\alpha . m) \bar{E}(\text { res })} \text { if } S_{i} \text { is advanced node }
\end{array}\right.
$$

The T $\left(S_{i}\right)$ probability threshold by which node $S_{i}$ uses to determine whether it can be elected as $\mathrm{CH}$ in a round is defined as:

$$
\mathrm{T}\left(S_{i}\right)=\left\{\begin{array}{cl}
\frac{p_{i}}{1-p_{i}\left(r \bmod 1 / p_{i}\right)} & \text { if } S_{i} \in R^{\prime} \\
0 & \text { otherwise }
\end{array}\right.
$$

Where $R^{\prime}$ is defined as the set of either normal or advance nodes that are not elected as CHs within the last $1 / p_{\text {nrml }}$ or $1 / p_{\text {adv }}$ rounds.

Proposed ZEEP: In this work, a novel Zonal based Energy Efficient Routing Protocol (ZEEP) for Heterogeneous WSN has been proposed in order to enhance the stability period, network lifetime and throughput of the network. In ZEEP, total area is partitioned into fixed number zones and deployment of different types of nodes in different zones according to their initial energy in order to provide the efficient utilization of total coverage area. Sensor nodes transmit the data to associated $\mathrm{CH}$ by using single-hop intra-cluster communication while CHs use short distance single-hop inter-cluster communication to transmit the data to BS. ZEEP uses hybrid routing approach for transmitting data to base station in which some sensor nodes transmit data directly to base station while other use 
clustering techniques to transmit the data to base station. ZEEP uses residual energy based election of CHs double layered election probability mechanism.

\section{Simulation and Results Analysis}

In this section, we evaluate the performance of purposed routing scheme. The following performance parameters are used to evaluate the performance of purposed routing scheme.

Stability Period. Stability Period is the time interval from when the network operation starts until death of first node.

Instability Period. Instability Period is the time interval starting from the death of $1^{\text {st }}$ node till death of last node.

Network Lifetime. Network lifetime is the time interval between the network operation start until the death of last node.

Throughput. This will measure the total number of packets which are sent to base station.

Network Remaining Energy. It will measure the total remaining energy of the network.

We compare it with LEACH and SEP in the same heterogeneous setting. The packet size is 500 bytes and initial energy is $E_{0}=0.5 \mathrm{~J}$. The other key parameters used in simulation are given in Table 1 .

Table 1 Simulation Parameters

\begin{tabular}{cl}
\hline Parameter & Value \\
\hline Area & $100 \mathrm{~m} \times 100 \mathrm{~m}$ \\
$N$ & 100 \\
$E_{\text {elec }}$ & $50 \mathrm{~nJ} / \mathrm{bit}$ \\
$E_{D A}$ & $5 \mathrm{~nJ} / \mathrm{bit} / \mathrm{message}$ \\
$E_{m p}$ & $10 \mathrm{pJ} / \mathrm{bit} / \mathrm{m}^{4}$ \\
$E_{f s}$ & $0.0013 \mathrm{pJ} / \mathrm{bit} / \mathrm{m}^{2}$ \\
$P_{o p t}$ & 0.1 \\
$d_{o}$ & $87.7 \mathrm{~m}$ \\
\hline
\end{tabular}

We performed the simulations for $\alpha=2, \mathrm{~m}=0.1$ and rounds $=5000$. According to Table 2, we can see that ZEEP has enhanced the stability period and network lifetime as compared to LEACH and SEP. In the LEACH and SEP, the death of first node occurs at $563^{\text {rd }}$ round and $879^{\text {th }}$ round respectively while in ZEEP first node died at $1125^{\text {th }}$ round. In LEACH and SEP all nodes died at $2301^{\text {st }}$ round and $2465^{\text {th }}$ round respectively while in ZEEP all nodes died after 4919 rounds. We can verify that the stability period and network lifetime is improved in the case of ZEEP as compare to LEACH and SEP.

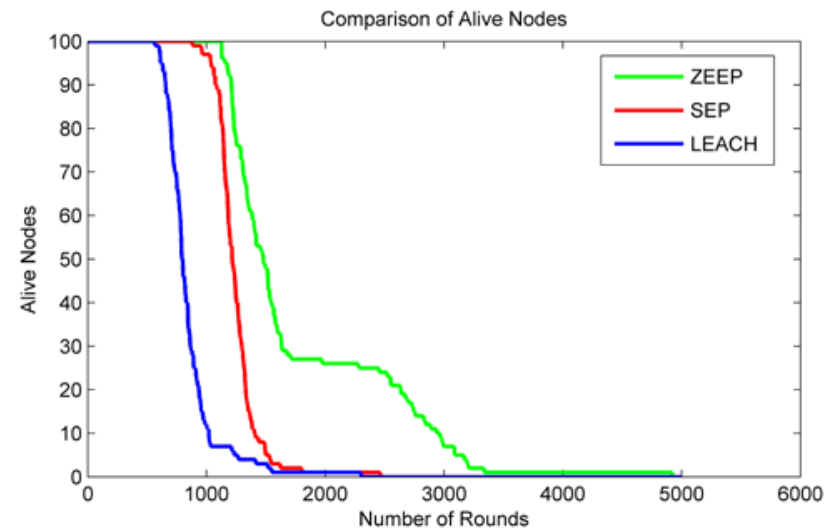

Fig. 1. Comparison of Alive Nodes

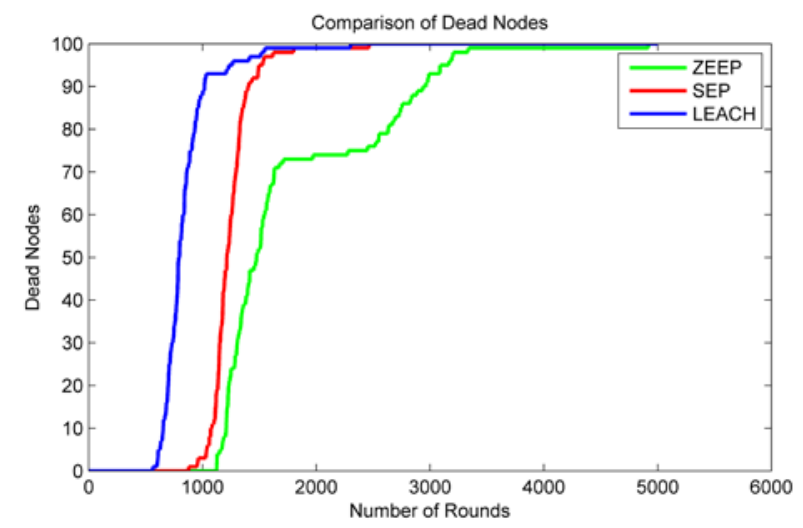

Fig. 2. Comparison of Dead Nodes

Fig. 1 shows that the stability period of ZEEP is more than that of LEACH and SEP. Fig. 2 shows the instability period of ZEEP is more than that of LEACH and SEP. Thus, it is clearly concluded that stability period and network lifetime is enhanced in the case of ZEEP as compared to LEACH and SEP. In Table 2, we can see that ZEEP has enhanced the throughput of the network as compared to LEACH and SEP. The throughput of LEACH and SEP is less because only CH transmits data to BS. 
Table 2 Comparison Table

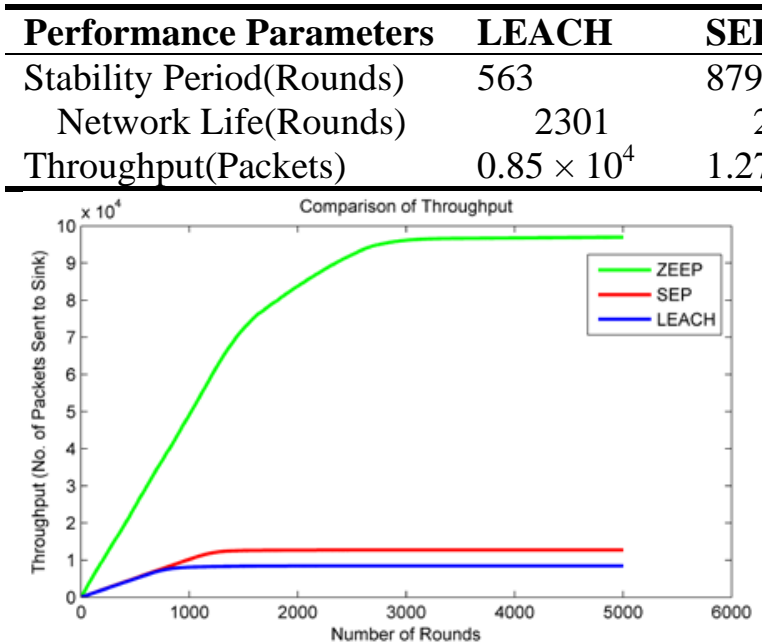

Fig. 3. Comparison of Throughput

$\begin{array}{cc}\text { SEP } & \text { ZEEP } \\ 879 & 1125 \\ 2465 & 4919 \\ 1.27 \times 10^{4} & 9.69 \times 10^{4}\end{array}$

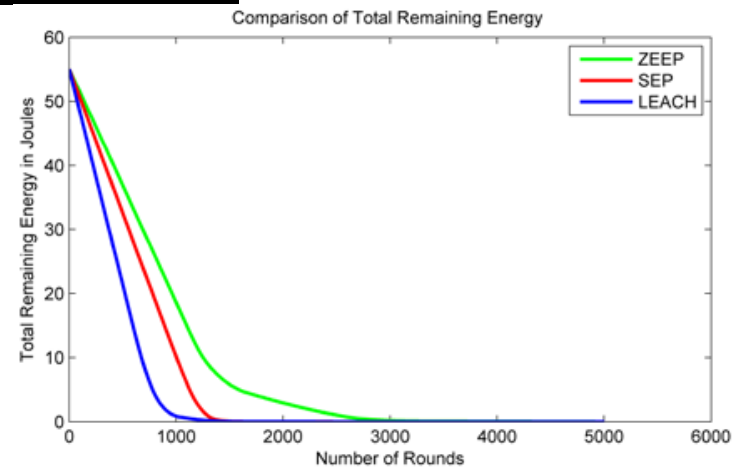

Fig. 4. Comparison of Total Remaining Energy

Fig. 3 shows the throughput of ZEEP is higher than LEACH and SEP. The results show that the total number of packets sent from CHs to sink is higher in the case of ZEEP as compared to LEACH and SEP. Our simulation results prove that the throughput of ZEEP is higher than LEACH and SEP. Fig. 4 shows the throughput of ZEEP is higher than LEACH and SEP. In Fig. 4, we can observe that total remaining energy in joules is more in ZEEP as compared to LEACH and SEP. The results show the total remaining energy in joules of ZEEP is higher than LEACH and SEP.

\section{Conclusion}

The simulation results clearly proved that the purposed routing protocol, ZEEP, significantly enhanced the stability period and network life time and throughput of the sensor network. ZEEP partitions the sensing region into zones to provide efficient utilization of total coverage area. ZEEP uses short distance energy efficient intra-cluster and inter-cluster communication approach in order to reduce the energy consumption. In future, ZEEP can be extended to incorporate more types of nodes.

\section{References}

[1] I.F. Akyildiz, W.Su, Y. Sankarasubmmaiam, E.Cayirci, “A Survey on Sensor Networks,” IEEE Comm. Magazine, pp.102-114, Aug 2002.

[2] R Kumar, V. Tsiatsis, M.B. Srivastava, Computation Hierarchy for In-Network Processing, Proceedings of 2nd ACM International Workshop on Wireless Networks and Applications, San Diego, CA, 68-77, 2003.

[3] Kemal Akkaya and Mohamed Younis, "A Survey on Routing Protocols for Wireless Sensor Networks,” Ad Hoc Networks, Vol. 3, No. 3, pp. 325-349, May 2005.

[4] J. Al-Karaki, and A. Kamal, "Routing Techniques in Wireless Sensor Networks: A Survey," IEEE Communications Magazine, Vol. 11, no. 6, Dec. 2004, pp. 6 -28.

[5] W. Heinzelman, A. Chandrakasan, and H. Balakrishnan, "EnergyEfficient Communication Protocols for Wireless Microsensor Networks,” Proc. Of 33th Hawaiian International Conference on Systems Science, 2000.

[6] Smaragdakis, I Matta, A Bestavros. SEP: A Stable Election Protocol for clustered heterogeneous wireless sensor networks. in: Second International Workshop on Sensor and Actor Network Protocols and Applications (SANPA). 2004. 